\title{
Évaluation des transferts entre barrage et aquifères par la méthode de bilan d'une retenue en zone semi-aride. Cas d'El Haouareb en Tunisie centrale Transfer evaluation between dam and aquifers by the balance
method in semi-arid region
}

\author{
A. Kingumbi, M. Besbes, J. Bourges et P. Garetta
}

Volume 17, numéro 2, 2004

URI : https://id.erudit.org/iderudit/705531ar

DOI : https://doi.org/10.7202/705531ar

\section{Aller au sommaire du numéro}

\section{Éditeur(s)}

Université du Québec - INRS-Eau, Terre et Environnement (INRS-ETE)

ISSN

0992-7158 (imprimé)

1718-8598 (numérique)

Découvrir la revue

Citer cet article

Kingumbi, A., Besbes, M., Bourges, J. \& Garetta, P. (2004). Évaluation des transferts entre barrage et aquifères par la méthode de bilan d'une retenue en zone semi-aride. Cas d'El Haouareb en Tunisie centrale. Revue des sciences de l'eau / Journal of Water Science, 17(2), 213-225. https://doi.org/10.7202/705531ar
Résumé de l'article

Le barrage d'El Haouareb est situé en Tunisie centrale, dans une zone caractérisée par un climat semi-aride. Il draine le bassin versant de l'oued Merguellil d'une superficie d'environ $1200 \mathrm{~km} 2$. Le but de cette recherche est d'évaluer, à partir du bilan du barrage, l'impact de la mise en place de cet ouvrage sur les ressources en eau de surface et souterraine, en vue de mieux cerner le fonctionnement de l'ensemble de ce système et faciliter ainsi sa gestion.

Le bilan en eau de surface fait appel à deux termes non mesurés : l'infiltration à travers la cuvette de la retenue et les apports des oueds incomplètement contrôlés. Par une méthodologie originale, nous avons modélisé et calculé l'infiltration. Les apports journaliers au barrage, déduits du bilan ont été ensuite validés par comparaison aux débits mesurés sur une station située, plus en amont, sur le tributaire principal.

Cette recherche a mis en évidence que, malgré des pertes non négligeables par évaporation ( $25 \%$ des apports), l'infiltration constitue, de loin, le terme le plus important du bilan de la retenue d'El Haouareb (63\% des apports).

Cette dernière a ainsi injecté, directement ou par nappe interposée, dans l'aquifère aval de la plaine de Kairouan plus de 9 millions de $\mathrm{m} 3$ par an. 


\title{
Évaluation des transferts entre barrage et aquifères par la méthode de bilan d'une retenue en zone semi-aride. Cas d'El Haouareb en Tunisie centrale
}

\author{
Transfer evaluation between dam and aquifers \\ by the balance method in semi-arid region. \\ The El Haouareb case in Central Tunisia
}

\section{A. KINGUMBI ${ }^{1 *}$, M. BESBES ${ }^{2}$, J. BOURGES ${ }^{3}$, P. GARETTA ${ }^{4}$}

Reçu le 19 septembre 2002, accepté le 22 mai 2003**.

\section{SUMMARY}

\begin{abstract}
The El Haouareb dam and the neighbouring dams (Nebhana and Sidi Saâd) were constructed with the objective of protecting the inhabitants of the Kairouan plain from floods. This area constitutes the natural downstream region of three principal wadis of central Tunisia (Merguellil, Nebhana and Zeroud) where there have been floods in the past. The second objective of this dam is to control the recharge of the Kairouan groundwater aquifer in the absence of natural wadi flows, by diverting flows from the dam and by infiltration through the foundation and basin of the dam. In a semi-arid region characterized by very strong evaporation and plant transpiration rates (about 1700 mm per year), it is obviously of great importance to recharge the underground water systems, which are less subject to these kinds of losses and which better preserve the water resources. The last objective of this dam is to supply water to the population, especially for agricultural needs. The dam was expected to supply water to an irrigated area of about 2500 hectares in this region. During the construction of the El Haouareb dam spillway, underground flows occurred in the limestone of the El Haouareb Hill. At the beginning of the exploitation of the dam these underground flows increased. This development has affected the management plans for the dam and accordingly a monitoring program has been put in place to follow of the evolution over
\end{abstract}

1 École Nationale d'Ingénieurs de Tunis (ENIT), Laboratoire d'Hydraulique, BP 37, 1002 Tunis. Tél. 00216 71874700 , Fax 0021671872729 , E-mail : kingumbi@yahoo.fr.

2 École Nationale d'Ingénieurs de Tunis (ENIT), Laboratoire d'Hydraulique, BP 37, 1002 Tunis. Tél. 00216 71874700 , Fax 0021671872729 , E-mail : mbt.besbes@gnet.tn.

3 Institut de Recherche pour le Développement (IRD) Mission Tunisie, BP 434, 1004 El Menzah IV Tunis. Tél. 0021671750 009, fax 0021671750254 , E-mail : jack.bourges@libertysurf.fr.

4 Institut de Recherche pour le Développement (IRD) Mission Tunisie, BP 434, 1004 El Menzah IV Tunis. Tél. 0021671750009 , fax 0021671750254 , E-mail : Philippe.Garetta@mpl.ird.fr.

** Les commentaires seront reçus jusqu'au 30 décembre 2004. 
time of the infiltration. The purpose of this research was to evaluate, by the water balance method, the impact of the construction of this dam on surface and ground water. This research will improve the understanding of the system, and thus, facilitate management. In addition, this project will evaluate recharge rates towards the groundwater reserves of the Kairouan plain, which constitutes the natural downstream region of this system.

The dam is situated at an interface of several superficial and underground hydraulic systems. For surface water, the El Haouareb dam drains the Merguellil wadi catchment whose surface area is about $1200 \mathrm{~km}^{2}$. Besides the Merguellil wadi, two of its tributaries flow directly into the El Haouareb dam (Hammam and Ben Zitoune wadis) but without any measurement of their flows. Nevertheless, two hydrometric stations were established on the Merguellil wadi, upstream of El Haouareb dam: Haffouz in 1966 and Sidi Boujdaria in 1974. The first station is still functional whereas the second one stopped working in 1989. For underground systems, the Ain Baïdha groundwater located upstream from the dam, and the downstream groundwater of the Kairouan plain constitute the principal aquifers in relation to this dam. A third aquifer of the El Haouareb hill, composed mainly of limestone, was also identified. It receives both Aïn Baïdha groundwater and infiltrations from the El Haouareb dam, and empties towards the groundwater of the Kairouan plain. A daily database of El Haouareb dam management, collected by the agricultural ministry, was available. The data-base contained the following elements: rainfall amounts for the site; water levels in the dam; evaporation rates measured by a Colorado tub; three states of the physical characteristics of dam measured before the beginning of exploitation (1989) and during two drying times of the dam (1994 and 1997); releases from the dam for irrigation; releases from the dam to fight against siltation of the dam and to recharge the Kairouan plain aquifer. There were also rain data measured from the surrounding rain gages and hydrometric data observed at the Haffouz and Sidi Boujdaria stations. The water balance equation of this dam presents two unknowns: the infiltration from the dam and the flows that arrive in the dam by wadis. To resolve these unknowns, we proceeded by minimizing one of the two unknowns with a goal to determine the other one. As infiltration rates were inaccessible, we worked on periods without flows, chosen according to four criteria. During these periods the rain on the dam and on the surrounding rain gages must be equal to $0 \mathrm{~mm}$, the flows measured at the upstream station of Haffouz must be inferior or equal to $50 \mathrm{~L} / \mathrm{s}$, and the variation of the water level in the dam must always be decreasing. Finally, the fourth criterion is that the length of these periods that must be equal or greater than $15 \mathrm{~d}$. Thus we were able to calculate the infiltration flows during the periods without flows. These values were corrected by adding the low water flows, which were assumed to arrive at the dam. These flows were calculated by a linear equation established between the low water flows at the Haffouz and Sidi Boujdaria stations. The representation of this calculated infiltration according to the water level in the dam gave a cluster of points from which three simple linear adjustments were done. Thus, we obtained a model composed of three linear equations that represented the changes in infiltration as a function of time. This model showed that the infiltration flow rate, corresponding to a particular level, decreased with time as sedimentation in the dam proceeded. For the daily average level $(206$ meters), the infiltration rate passed from $650 \mathrm{~L} / \mathrm{s}$ in 1989 to $310 \mathrm{~L} / \mathrm{s}$ in 1998 . Despite the scarcity of "periods without flows" between 1989 and 1991, and some uncertainties due essentially to the measurement of water levels in the dam, this model is representative of reality because it takes into account the evolution of sedimentation in the dam. The model yielded infiltration flow rates that were higher than those calculated in preceding studies. The infiltration $m$ sdel obtained was then applied to the entire period of study, allowing resc'ution of the water balance equation for the dam (which now has only one unknown): the flows of the different wadis arriving in the dam. 
In conclusion, this research showed that despite considerable losses by evaporation ( $25 \%$ of flows), infiltration (accounting for $63 \%$ of flows) constituted the more important term in the water balance of the El Haouareb dam. This dam has thus injected, directly or via the interposed limestone aquifer, more than 9 million $\mathrm{m}^{3}$ per year into the downstream groundwater of the Kairouan plain.

Key words : El Haouareb dam, balance method, infiltration, underground flows, aquifer, semi-arid region.

\section{RÉSUMÉ}

Le barrage d'El Haouareb est situé en Tunisie centrale, dans une zone caractérisée par un climat semi-aride. Il draine le bassin versant de l'oued Merguellil d'une superficie d'environ $1200 \mathrm{~km}^{2}$. Le but de cette recherche est d'évaluer, à partir du bilan du barrage, l'impact de la mise en place de cet ouvrage sur les ressources en eau de surface et souterraine, en vue de mieux cerner le fonctionnement de l'ensemble de ce système et faciliter ainsi sa gestion.

Le bilan en eau de surface fait appel à deux termes non mesurés : l'infiltration à travers la cuvette de la retenue et les apports des oueds incomplètement contrôlés. Par une méthodologie originale, nous avons modélisé et calculé l'infiltration. Les apports journaliers au barrage, déduits du bilan ont été ensuite validés par comparaison aux débits mesurés sur une station située, plus en amont, sur le tributaire principal.

Cette recherche a mis en évidence que, malgré des pertes non négligeables par évaporation ( $25 \%$ des apports), l'infiltration constitue, de loin, le terme le plus important du bilan de la retenue d'El Haouareb (63\% des apports). Cette dernière a ainsi injecté, directement ou par nappe interposée, dans l'aquifère aval de la plaine de Kairouan plus de 9 millions de $\mathrm{m}^{3}$ par an.

Mots clés : barrage El Haouareb, méthode de bilan, infiltration, écoulements souterrains, aquifere, région semi-aride.

\section{1 - INTRODUCTION}

Le barrage d'El Haouareb, situé dans le gouvernorat de Kairouan (figure 1), est à l'exutoire du bassin versant de l'oued Merguellil d'une superficie d'environ $1200 \mathrm{~km}^{2}$ (KINGUMBI, 1997). À la mise en eau de ce barrage (1989) son stockage a été évalué à 95 millions de $\mathrm{m}^{3}$ à la cote de sa retenue normale $(217 \mathrm{~m})$ et à 212 millions de $\mathrm{m}^{3}$ à sa plus haute cote $(225 \mathrm{~m})$. Il a été construit pour remplir trois principaux objectifs. Le premier se rapporte à la protection contre les inondations qui ont causé, à plusieurs reprises, des dommages dans la plaine de Kairouan. Le deuxième objectif concerne la satisfaction des besoins en eaux des populations environnantes dans un contexte semi-aride où cette ressource est rare. C'est ainsi qu'un périmètre irrigué d'une superficie d'environ 2500 ha a été prévu dans la zone d'El Haouareb. Le dernier objectif consiste en la recharge de la nappe de la plaine de Kairouan en absence des écoulements naturels des oueds. L'alimentation des nappes est particulièrement importante dans ce contexte climatique caractérisé par une très forte évapotranspiration (ETP $\approx$ $1700 \mathrm{~mm} / \mathrm{an}$ ) puisqu'elle permet ainsi de conserver les ressources en eaux. 


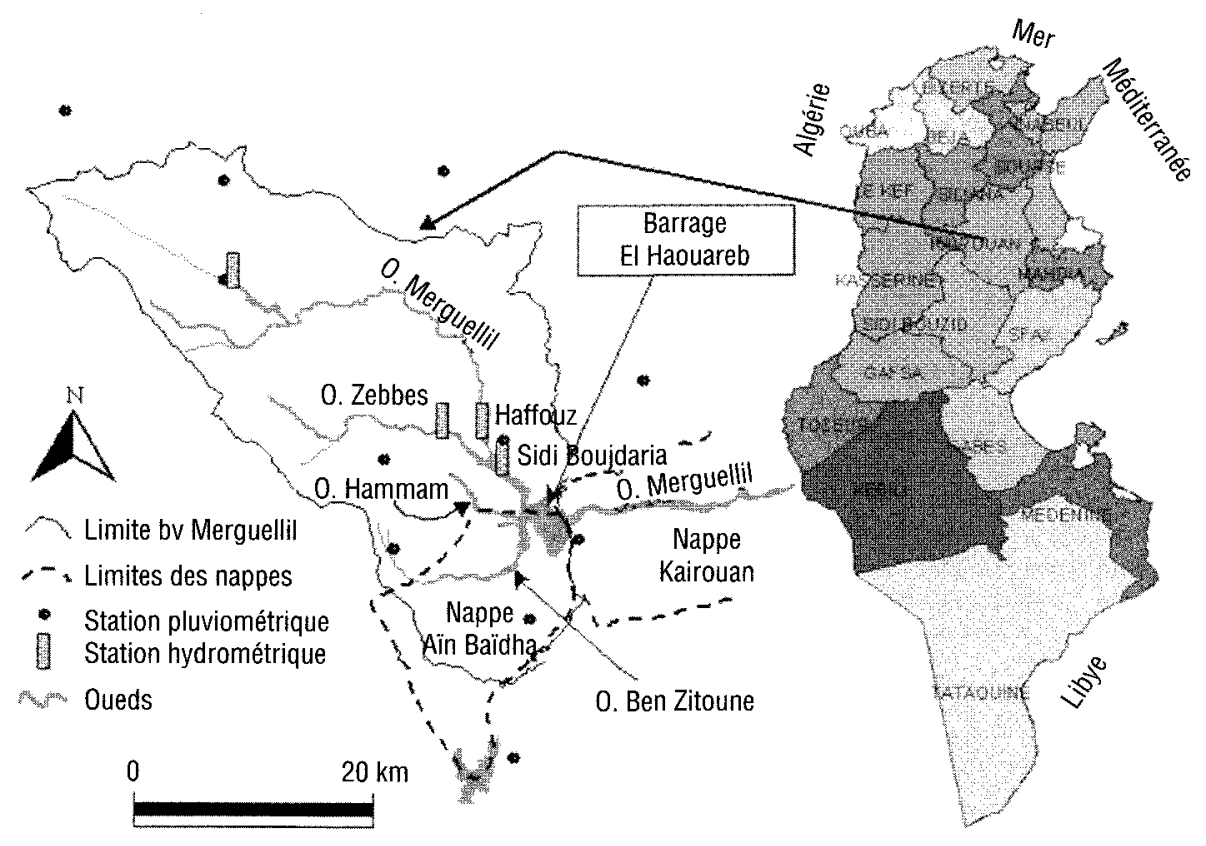

Figure 1 Présentation et situation de la zone d'étude.

Presentation and localisation of zone of study.

L'oued Merguellil draine le gros des apports qui arrivent au barrage. Deux autres oueds (Hammam et Ben Zitoune), de moindre importance, se déversent aussi dans le barrage sans aucun dispositif de contrôle d'apports à l'entrée (figure 1). Quelques stations hydrométriques ont, cependant, été implantées à l'amont immédiat du barrage depuis la fin des années soixante. II s'agit principalement des stations de Haffouz et Sidi Boujdaria, implantées respectivement en 1965 et en 1974. Les mesures hydrométriques continuent sur la première alors que la seconde a cessé de fonctionner depuis la mise en eau du barrage.

Sur le plan souterrain, le barrage d'El Haouareb se trouve à l'interface de deux importants systèmes aquifères : la nappe d'Aïn Baïdha et celle de la plaine de Kairouan. La première se trouve à l'amont de la retenue et ses flux convergent vers le seuil d'El Haouareb par lequel ils alimentent la nappe de la plaine de Kairouan (BABASY, 1999 ; HAMZA, 1976) qui constitue l'exutoire naturel de tout le système (superficiel et souterrain) amont.

Après la mise en eau du barrage d'El Haouareb, ces nappes ont enregistré une remontée piézométrique dans les zones d'influence du barrage. La figure 2 montre une augmentation du niveau piézométrique de 2 et 8 mètres respectivement dans la nappe d'Aïn Baïdha et celle de la plaine de Kairouan. Une remontée équivalente a cependant été observée en 1969 dans la région. Elle est consécutive aux pluies exceptionnelles de cette année qui sont en moyenne sur le bassin du Merguellil deux fois supérieures à celles enregistrées en 1989. 


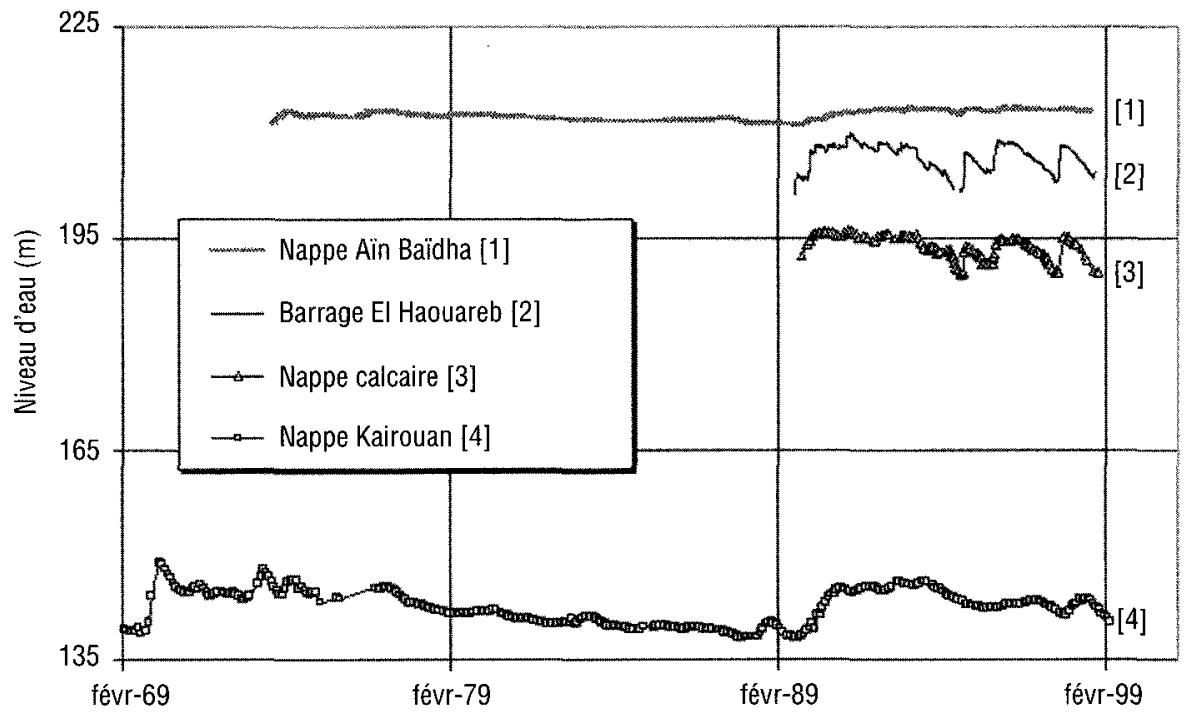

Figure 2 Niveaux d'eau des réservoirs dans la zone d'El Haouareb.

Water levels of different reservoirs in relation to El Haouareb Zone.

D'autre part, CHADLY (1992) a mis en évidence l'existence d'un aquifère intermédiaire dans les calcaires turoniens du djebel El Haouareb qui serait en communication avec le barrage (figure 2).

Pendant la construction de l'évacuateur des crues de ce barrage des émergences d'eau ont fait leur apparition. L'injection de ciment dans les formations fissurées a permis de baisser leur débit et de parachever les travaux. Ces émergences, qui ont continué de couler après la mise en eau du barrage, sont contrôlées par des mesures hebdomadaires.

Cette recherche a pour objectif de faire le bilan en eau de l'ensemble du système d'El Haouareb (souterrain et de surface) en vue d'évaluer le transfert entre le barrage et les différentes nappes en communication. Elle devrait aussi permettre de mieux comprendre le fonctionnement de ce système en vue de sa meilleure gestion.

\section{2 - DONNÉES}

Une banque de données sur le fonctionnement du barrage d'El Haouareb a été mise à notre disposition par la Direction Générale des Grands Travaux Hydrauliques (DGGTH). Elles concernent les mesures journalières de niveaux d'eau dans la retenue de ce barrage (figure 3), ses caractéristiques physiques (courbes hauteur surface volume), les données sur sa gestion et les données 
météorologiques. Les caractéristiques physiques (figure 4) évoluent, à cause de l'envasement du barrage, en fonction du temps et nous ne disposons que des résultats de trois campagnes de mesures (GARETTA et OUR GHEMI, 1999) que nous avons réparties sur toute la période d'étude (figure 3 ).

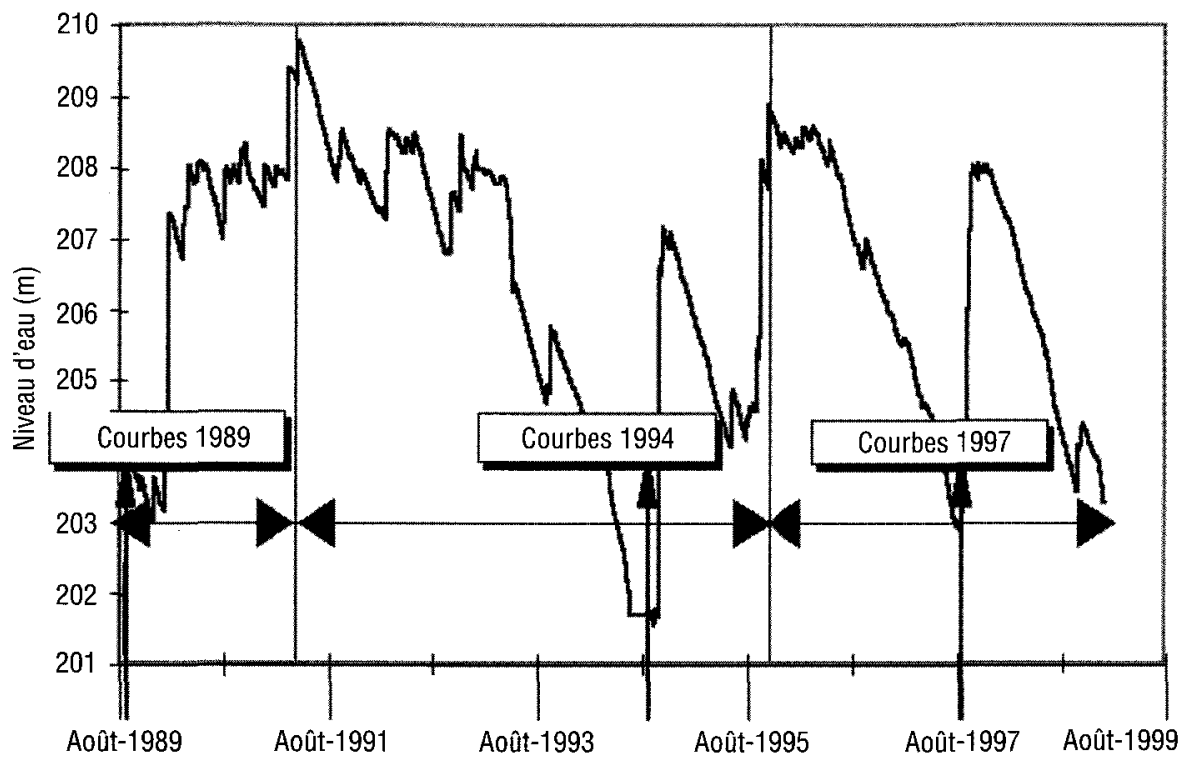

Figure 3 Niveau journalier d'eau dans le barrage d'El Haouareb.

Daily water level in El Haouareb dam.

En ce qui concerne la gestion du barrage, il y a les données des vidanges de fond, des lâchures pour l'alimentation de la nappe de Kairouan et des pompages dans la retenue pour l'irrigation du périmètre d'El Haouareb.

Une station météorologique comportant un bac d'évaporation, un pluviomètre, une girouette et un anémomètre a été mis en place avec la mise en eau du barrage. D'autres stations pluviométriques ont été implantées par la Direction Générale des Ressources en Eau (DGRE) dans la région depuis fort longtemps (figure 1).

Les lacunes dans les données pluviométriques ont été complétées en utilisant les stations voisines. En ce qui concerne l'évaporation, un coefficient a été appliqué pour tenir compte du transfert d'échelle entre le bac et le lac. Ce coefficient, déterminé en tenant compte non seulement des études faites par ailleurs (AL-TURBAK et AL-MUTTAIR, 1989 ; HAYOUNI, 1999 ; POUYAUD, 1986), mais aussi des directions saisonnières des vents dominants en Tunisie centrale (BOUZAIANE et LAFFORGUE, 1986), a été en moyenne estimé égal à 0,8. 

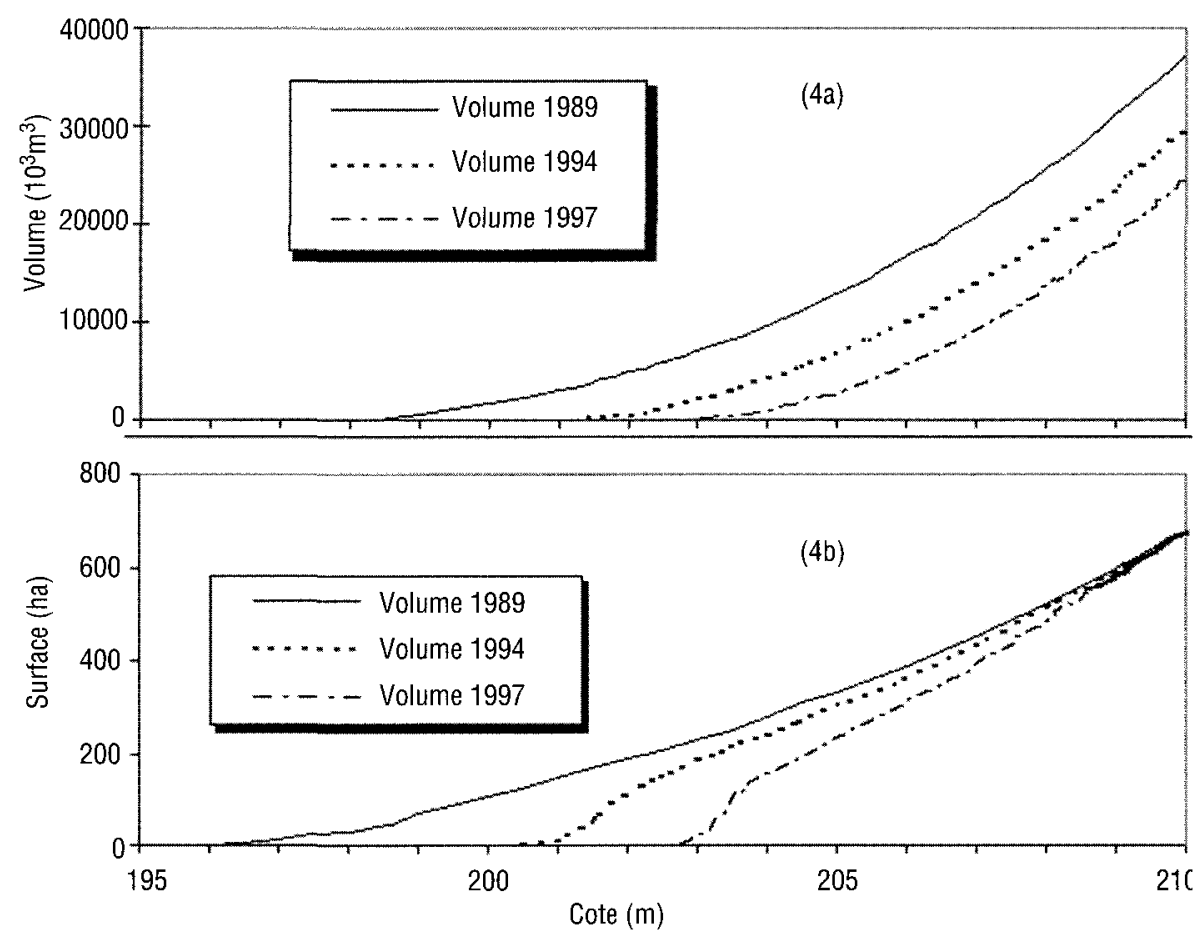

Figure 4 Évolution des caractéristiques physiques du barrage d'El Haouareb.

Physical characteristics evolution of El Haouareb dam.

\section{3 - MÉTHODOLOGIE}

La méthodologie adoptée se base sur l'équation de bilan d'une retenue, valable aussi bien sur les petits barrages (Direction CES et ORSTOM, 1996) que sur de grandes étendues d'eau (SHARON et al., 2000). Appliquée sur une retenue quelconque, pendant un intervalle de temps $\mathrm{dt}$, cette équation se présente comme suit :

$$
\left(V_{e}+V_{e c s}+V_{p}\right)-\left(V_{e v}+V_{v i}+V_{u}+V_{\text {inf }}+V_{d}\right)=\Delta V
$$

où $V_{e}$ est l'apport des oueds à la retenue, $V_{\text {ecs }}$ l'apport des nappes en relation avec la retenue, $V_{p}$ l'apport des précipitations sur la retenue, $V_{e v}$ le volume évaporé de la retenue, $V_{v i}$ le volume vidangé de la retenue, $V_{u}$ le volume sortie de la retenue pour différentes utilisations, $V_{\text {inf }}$ le volume infiltré de la retenue, $V_{d}$ le volume déversé, et $\Delta V$ la variation du stock dans la retenue.

Appliquée à la retenue d'El Haouareb, cette équation s'est révélée présenter deux inconnues: les apports des oueds au barrage et la résultante des échanges entre la retenue et le système souterrain :

$$
V_{e}-V_{i}=\Delta V+V_{e v}+V_{v i}+V_{u}-V_{p}
$$


où $V_{i}=V_{\text {ecs }}-V_{\text {inf }}$ représente, pendant l'intervalle $\mathrm{dt}$, les échanges entre la retenue et le système souterrain.

L'équation de bilan du barrage d'El Haouareb (2) admet une infinité de solutions. Pour la résoudre, il faut minimiser l'une des deux inconnues en vue de déterminer l'autre. Comme l'infiltration est inaccessible, nous avons minimisé les apports au barrage. Nous avons ainsi travaillé sur des périodes dites sans apports choisies en fonction de leur durée d'au moins 15 jours et durant lesquelles aucune pluie n'a été signalée ni sur le barrage et ni dans ses environs. Au cours de ces périodes, les débits mesurés à la station amont de Haffouz devaient être inférieurs à $50 \mathrm{l} / \mathrm{s}$ et la variation de niveau d'eau dans la retenue toujours décroissante.

Nous avons ainsi obtenu, dans la première approximation, l'équation de bilan du barrage d'El Haouareb correspondant aux périodes sans apports :

$$
V_{i}=-\left(\Delta V+V_{e v}+V_{v i}+V_{u}\right)
$$

Mais comme les apports ne sont jamais nuls, dans la deuxième approximation nous avons tenu compte des apports mesurés à la station de Haffouz. Pour cela, une corrélation entre les débits d'étiages de la station de Haffouz et ceux de la station de Sidi Boujdaria, qui sont supposés se propager jusqu'au barrage d'El Haouareb, a été établie (éq. 4) avec un coefficient de corrélation égal à 0,88 .

$$
\text { Débit }(/ / s)_{\text {Sidi Boujdaria }}=1,39^{\star} \text { Débit }(1 / s)_{\text {Haffouz }}+35.5
$$

Nous avons ainsi calculé, pendant les périodes sans apports, l'exfiltration journalière de la retenue d'El Haouareb, que nous avons modélisé en fonction de la cote d'eau dans la retenue.

Le modèle d'infiltration ainsi obtenu a été étendu sur l'ensemble de la période d'étude, ce qui nous a permis d'estimer tous les éléments du bilan en eau de surface de cette retenue.

Le bilan en eau souterraine a été établi au niveau de l'aquifère des calcaires turoniens d'El Haouareb. Sans capacité de stockage significatif, cet aquifère a été considéré comme une zone de transit des eaux provenant du barrage et de la nappe d'Aïn Baïdha qu'elle achemine vers la nappe aval de la plaine de Kairouan. Ceci a donné l'équation (5) du bilan en eau souterraine du système d'El Haouareb, admettant une seule inconnue que représente les percolations profondes vers la nappe de Kairouan.

$$
V_{i}+V_{A}=V_{E}+V_{P R}
$$

$V_{A}$ est le volume provenant du transfert de la nappe d'Aïn Baïdha, $V_{E}$ le volume sorti par les émergences à l'aval de l'évacuateur des crues, et $V_{P R}$ le volume percolé en profondeur vers la nappe de la plaine de Kairouan.

Le transfert de la nappe d'Aïn Baïdha a été calculé par le modèle mathématique de cette nappe élaboré par BABASY (1999) tandis que les sorties par les émergences ont été modélisées en fonction de la cote d'eau dans la retenue d'El Haouareb (figure 5). 


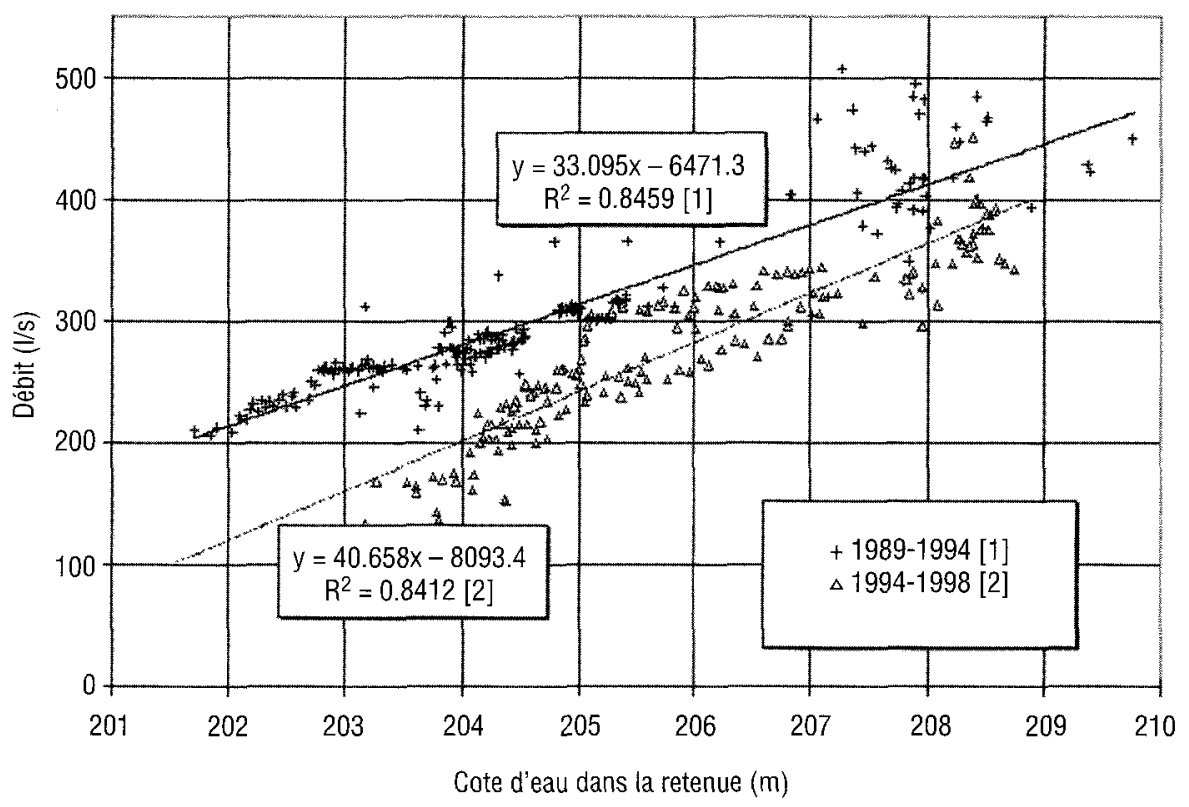

Figure 5 Débit d'émergence en fonction de la cote d'eau dans la retenue.

Underground flows vs. water level in El Haouareb dam.

\section{4 - RÉSULTATS}

Le modèle d'infiltration a été obtenu en représentant l'infiltration en fonction de la cote d'eau dans la retenue. Cette représentation a été faite de deux manières: la première a consisté à faire une moyenne sur une période sans apports (figure $6 \mathrm{a}$ ), alors que dans la seconde on a considéré pour une période sans apports les moyennes mobiles de 15 jours (figure 6b).

Nous avons ainsi obtenu un nuage de points sur lequel nous avons ajusté trois régressions linéaires (figure 6) qui montrent, pour une même cote, l'évolution décroissante de l'infiltration en fonction du temps.

Le modèle d'infiltration ainsi obtenu sur les périodes sans apports a été étendu sur l'ensemble de la période d'étude, ce qui fait que l'équation (2) de bilan du barrage d'El Haouareb s'est retrouvée avec une seule inconnue, les apports des oueds à la retenue. Ces derniers ont été calculés et comparés, pour validation, aux débits jaugés à la station de Haffouz située, plus en amont, sur l'oued Merguellil (figure 7).

Le bilan en eau du système d'El Haouareb (figure 8) met en exergue l'importance de l'infiltration dans le bilan superficiel de ce système. II montre qu'une bonne partie (environ $65 \%$ ) de ces infiltrations, 9 millions de $\mathrm{m}^{3}$ par an, a atteint la nappe de la plaine de Kairouan pendant les dix années d'exploitation du barrage. 
(6a) Moyenne par période sans apports

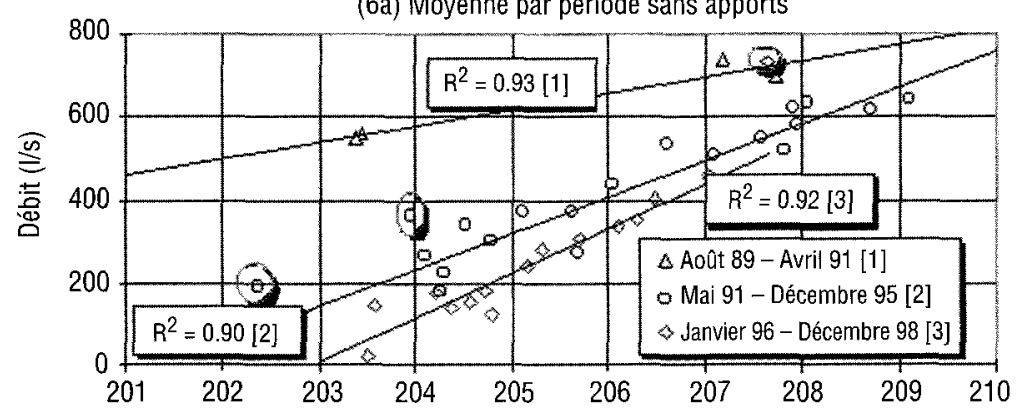

(6b) Moyennes mobiles de 15 jours

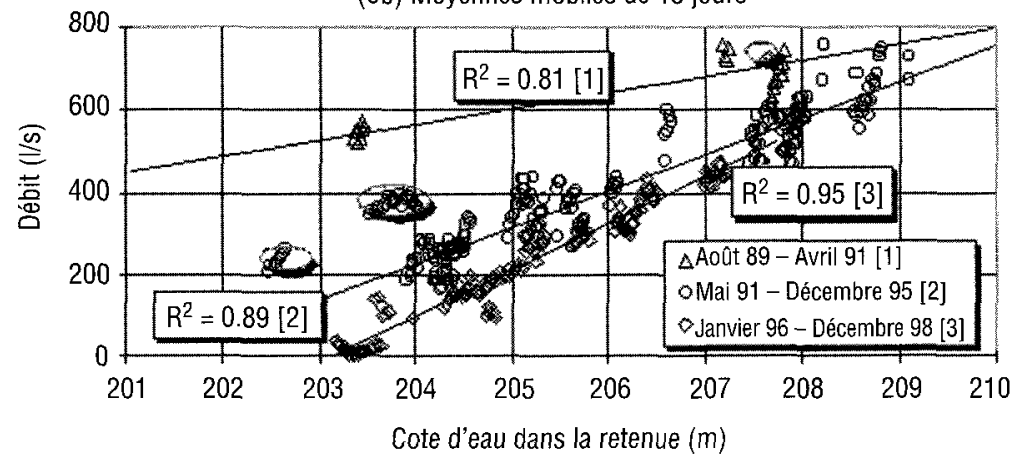

Figure 6 Débit infiltré en fonction de la cote d'eau dans la retenue d'El Haouareb. Infiltration flows vs, water level in El Haouareb dam.

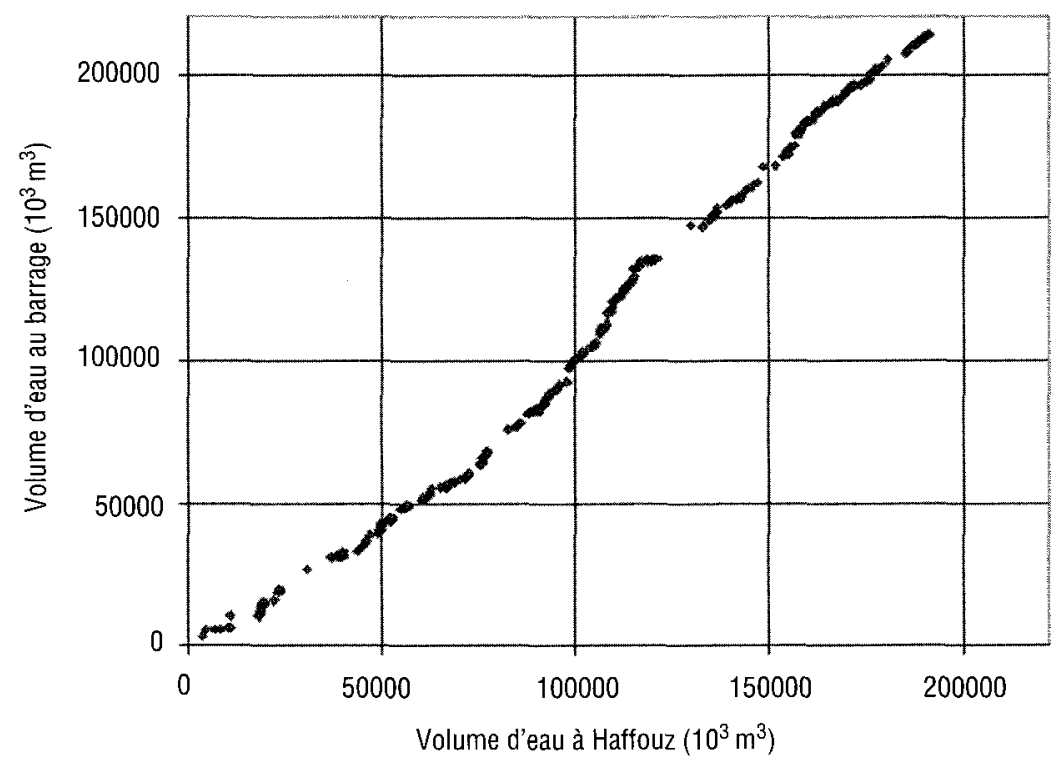

Figure 7 Comparaison du cumul des apports journaliers mesurés et calculés. Comparison of the some of measured and calculated daily runoff. 


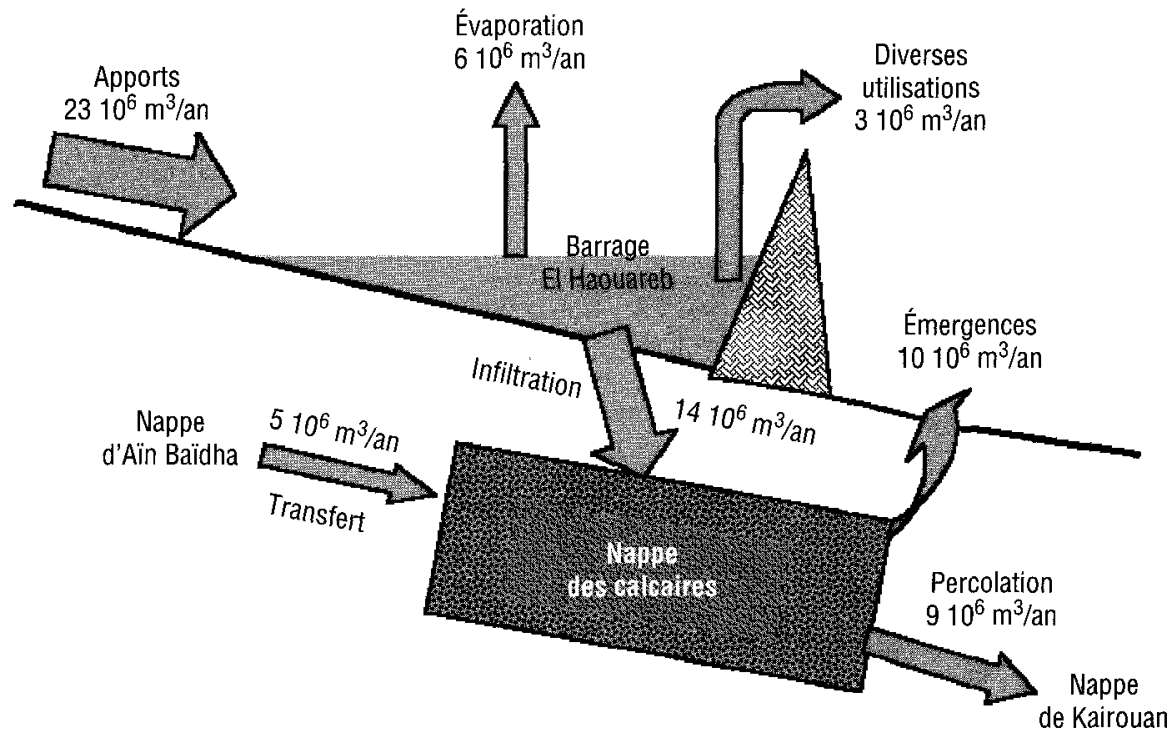

Figure 8 Bilan en eau du système d'El Haouareb pour ses dix premières années d'exploitation.

Water balance of El Haouareb system in first ten years.

\section{5 - DISCUSSIONS ET CONCLUSIONS}

Les deux méthodes de représentation de l'infiltration en fonction de la cote d'eau dans la retenue aboutissent, avec $2 \%$ de différence, aux mêmes résultats, ceci prouve que quinze jours représentent un délai assez suffisant pour dissiper l'incertitude des mesures journalières sur la valeur de l'infiltration.

Le modèle obtenu montre une évolution de l'infiltration en fonction du temps. En effet, comme on pouvait s'y attendre, au fur et à mesure que l'ouvrage se sédimente, il y a une tendance à la baisse de l'infiltration pour une même cote. Pour la cote journalière moyenne $(206 \mathrm{~m})$, elle passe de $650 \mathrm{l} / \mathrm{s}$ à la mise en eau (1989) à $310 \mathrm{l} / \mathrm{s}$ en 1998.

Dans ce modèle, trois périodes sans apports semblent produire des valeurs qui se décalent de l'allure générale des autres. Elles correspondraient aux périodes durant lesquelles des prélèvements clandestins d'eau auraient été effectués dans le barrage d'El Haouareb. D'ailleurs, deux de ces périodes coïncident avec l'assèchement de ce barrage en 1994.

Les infiltrations obtenues par cette méthode sont en grandeur moyenne supérieures de $60 \%$ aux prévisions de SMAOUI (1985) étant donné qu'il n'avait pas tenu compte, dans sa modélisation, des émergences d'eau à l'aval de l'évacuateur des crues. Ils se rapprochent par contre de ceux de IBRAHIM 
(1997) parce que ce dernier auteur a tenu compte de ces émergences, mais restent toujours supérieurs (de $20 \%$ en moyenne). Malgré le faible nombre de périodes sans apports entre la mise en eau et avril 1991, ces résultats sont plus représentatifs de la réalité puisqu'ils tiennent compte de l'évolution de la sédimentation du barrage d'El Haouareb. La précision de l'estimation de ce modèle, calculée en se basant essentiellement sur celle des mesures du niveau d'eau dans la retenue $(1 \mathrm{~cm})$, a été estimée à environ $10 \%$.

Étant donné que nous ne pouvons pas utiliser la cote d'eau dans la retenue, dont le fond change au fur et à mesure que l'ouvrage s'envase, dans un modèle de prévision à long terme de l'infiltration, il faut trouver une relation entre l'infiltration ainsi calculée et une variable caractéristique de la retenue plus permanente telle que le volume stocké (figure 9).

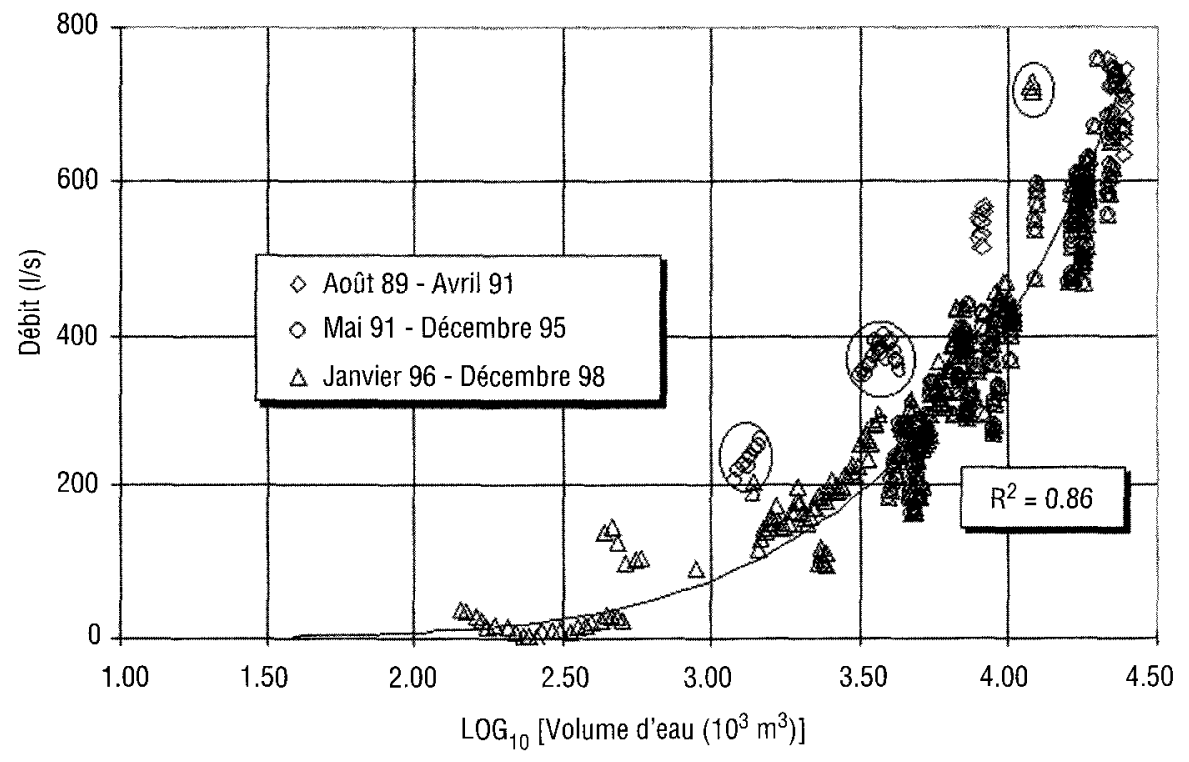

Figure 9 Débit infiltré en fonction du stockage dans la retenue d'El Haouareb. Infiltration flows vs. water volume stored in El Haouareb dam.

Les apports journaliers calculés au barrage d'El Haouareb par la méthode de bilan sont synchrones à ceux mesurés à Haffouz. Cependant, on trouve parfois des apports à cette station très supérieurs à ceux calculés au barrage, ce qui pose le problème de la qualité des mesures à la station de Haffouz connue pour l'instabilité de sa section pendant les crues.

Cette méthode peut être appliquée à d'autres retenues pour mieux cerner leurs bilans. Elle permettrait de mieux quantifier le phénomène d'infiltration, ce qui améliorerait la gestion de l'eau surtout dans les régions semi-arides où cette ressource est souvent très limitée. 


\section{RÉFÉRENCES BIBLIOGRAPHIQUES}

AL-TURBAK A. S., AL-MUTTAIR F. F., 1989. Evaluation of dams as a recharge method. Int. J. Water Resour. Dev., 5(2), 119-124.

BABASY L., 1999. Modélisation du système aquifère d'Aïn Baïdha. ENIT, Tunis, Tunisie, $65 \mathrm{p}$.

BOUZAIANE S., LAFFORGUE A., 1986. Monographie hydrologique des oueds Zeroud et Merguellil. DGRE - ORSTOM, Tunis, Tunisie, $1058 \mathrm{p}$.

CHADLY B., 1992. Note sur l'évolution de la nappe de Kairouan après la fermeture du barrage d'El Haouareb. DGRE, Tunis, Tunisie, $24 \mathrm{p}$.

DIRECTION CES, ORSTOM, 1996. Annuaire Hydrologique des lacs collinaires, 19941995, Tunis, Tunisie, $140 \mathrm{p}$.

GARETTA P., OUR GHEMI A., 1999. Résultats des nivellements de $1997 / 1998$ et mise à jour de la bathymétrie de la retenue d'El Haouareb. Évaluations des apports en sédiments. IRD - DG/EGTH, Tunis, Tunisie.

HAMZA M., 1976. Contribution à l'étude hydrogéologique du synclinal d'Aïn El Baïdha. Th. Doct. Univ. Paris VI, 171 p.
HAYOUNI S., 1999. Optimisation du système hydraulique du Kairouanais en vue d'un transfert d'eau. INAT, Tunis, Tunisie, $214 p$.

IBRAHIM A.N., 1997. Hydrologie du seuil d'El Haouareb avant et après la mise en eau du barrage. Étude sur modèle numérique. FST, Tunis, Tunisie, 57p.

KINGUMBI A., 1997. Caractérisation morphométrique du bassin versant du Merguellil. Application à la simulation des écoulements de surface et à l'érosion. IRD/E.S.I.E.R, Medjez El Bab, Tunisie, 59 p.

POUYAUD B., 1986. Contribution à l'évaluation de l'évaporation de nappes d'eau libre en climat tropical sec. Exemples du lac Bam et de la mare d'Oursi (BourkinaFaso), du lac Tchad et d'açudes du nord-est brésilien. Éditions ORSTOM, Bondy, $254 \mathrm{p}$.

SHARON E.N., XUNGANG Y., MAMOUDOU B.B., 2000. On the feasibility of using a lake water balance model to infer rainfall : an example from Lake Victoria. Hydrol. Sci. J., 45(1), 75-95.

SMAOUI M., 1986. Débits de fuite et gestion du barrage El Haouareb. ENIT, Tunis, Tunisie, $63 \mathrm{p}$. 